\title{
AN EXTENSION OF FIXED POINT THEOREMS CONCERNING CONE EXPANSION AND COMPRESSION AND ITS APPLICATION
}

\author{
Feng Wang and Fang Zhang
}

\begin{abstract}
The famous Guo-Krasnosel'skii fixed point theorems concerning cone expansion and compression of norm type and order type are extended, respectively. As an application, the existence of multiple positive solutions for systems of Hammerstein type integral equations is considered.
\end{abstract}

\section{Introduction and preliminaries}

There are many fixed point theorems. See [16] for an introduction to the study and applications of fixed point theorems. In this paper we will generalize the fixed point theorems concerning cone expansion and compression of norm type and order type.

Definition 1.1. Let $E$ be a real Banach space. A nonempty closed convex set $P \subset E$ is called a cone if it satisfies the following two conditions:

(i) $x \in P, \lambda \geq 0$ implies $\lambda x \in P$;

(ii) $x \in P,-x \in P$ implies $x=\theta$.

Every cone $P \subset E$ induces an ordering in $E$ given by

$$
x \leq y \text { if and only if } y-x \in P .
$$

Definition 1.2. An operator is called completely continuous if it is continuous and maps bounded sets into precompact sets.

Definition 1.3. Let $E$ and $E_{1}$ be two real Banach spaces with cones $P$ and $P_{1}$ respectively. Then the operator $B: P \rightarrow P_{1}$ is said to be homogeneous on $P$ provided any $t \in \mathbb{R}^{+}, x \in P$ implies $B(t x)=t B x$.

Received December 29, 2007.

2000 Mathematics Subject Classification. Primary 47H10, 34B10, 34B15.

Key words and phrases. fixed point theorems, cone expansion and compression, Hammerstein integral equations.

This work was financially supported by NNSF of China 10671167. 
Definition 1.4. Let $E$ and $E_{1}$ be two real Banach spaces with cones $P$ and $P_{1}$ respectively. Then the operator $B: P \rightarrow P_{1}$ is said to be order-preserving on $P$ provided $x_{1}, x_{2} \in P$ with $x_{1} \leq x_{2}$ implies $B x_{1} \leq B x_{2}$.

The following theorem, which establishes the existence and uniqueness of fixed point index, is from [5]; an elementary proof can be found in [3]. The proof of the generalization of the fixed point theorems of norm type and order type in the section will invoke the properties of the fixed point index. The proof of the following fixed point index results can be found in $[3,5]$.

Lemma 1.1. Let $X$ be a retract of a real Banach space $E$. Then, for every bounded relatively open subset $U$ of $X$ and every completely continuous operator $A: \bar{U} \rightarrow X$ which has no fixed points on $\partial U$ (relative to $X)$, there exists an integer $i(A, U, X)$ satisfying the following conditions:

$\left(G_{1}\right)$ Normality: $i(A, U, X)=1$ if $A x \equiv y_{0} \in U$ for any $x \in \bar{U}$;

$\left(G_{2}\right)$ Additivity: $i(A, U, X)=i\left(A, U_{1}, X\right)+i\left(A, U_{2}, X\right)$ whenever $U_{1}$ and $U_{2}$ are disjoint open subsets of $U$ such that $A$ has no fixed points on $\bar{U} \backslash\left(U_{1} \cup U_{2}\right)$;

$\left(G_{3}\right)$ Homotopy Invariance: $i(H(t, \cdot), U, X)$ is independent of $t \in[0,1]$ whenever $H:[0,1] \times \bar{U} \rightarrow X$ is completely continuous and $H(t, x) \neq x$ for any $(t, x) \in[0,1] \times \partial U$;

$\left(G_{4}\right)$ Permanence: $i(A, U, X)=i(A, U \cap Y, Y)$ if $Y$ is a retract of $X$ and $A(\bar{U}) \subset Y$

$\left(G_{5}\right)$ Excision: $i(A, U, X)=i\left(A, U_{0}, X\right)$ whenever $U_{0}$ is an open subset of $U$ such that $A$ has no fixed points in $\bar{U} \backslash U_{0}$;

$\left(G_{6}\right)$ Solution: If $i(A, U, X) \neq 0$, then $A$ has at least one fixed point in $U$. Moreover, $i(A, U, X)$ is uniquely defined.

Now we state the Guo-Krasnosel'skii fixed point theorems concerning cone expansion and compression of norm type and order type as follows (see $[3,5]$ ).

Theorem 1.1. Let $\Omega_{1}$ and $\Omega_{2}$ be two bounded open sets in $E$ such that $\theta \in \Omega_{1}$ and $\bar{\Omega}_{1} \subset \Omega_{2}$. Suppose that $A: P \cap\left(\bar{\Omega}_{2} \backslash \Omega_{1}\right) \rightarrow P$ is completely continuous. If one of the two conditions

$\left(H_{1}\right) \quad\|A x\| \leq\|x\|, \forall x \in P \cap \partial \Omega_{1}$ and $\|A x\| \geq\|x\|, \forall x \in P \cap \partial \Omega_{2}$ and

$\left(H_{2}\right) \quad\|A x\| \geq\|x\|, \forall x \in P \cap \partial \Omega_{1}$ and $\|A x\| \leq\|x\|, \forall x \in P \cap \partial \Omega_{2}$ is satisfied, then $A$ has at least one fixed point in $P \cap\left(\bar{\Omega}_{2} \backslash \Omega_{1}\right)$.

Theorem 1.2. Let $\Omega_{1}$ and $\Omega_{2}$ be two bounded open sets in $E$ such that $\theta \in \Omega_{1}$ and $\bar{\Omega}_{1} \subset \Omega_{2}$. Suppose that $A: P \cap\left(\bar{\Omega}_{2} \backslash \Omega_{1}\right) \rightarrow P$ is completely continuous. If one of the two conditions

$\left(H_{3}\right) \quad A x \nsupseteq x, \forall x \in P \cap \partial \Omega_{1}$ and $A x \not x x, \forall x \in P \cap \partial \Omega_{2}$ and

$\left(H_{4}\right) \quad A x \not \leq x, \forall x \in P \cap \partial \Omega_{1}$ and $A x \nsupseteq x, \forall x \in P \cap \partial \Omega_{2}$ is satisfied, then $A$ has at least one fixed point in $P \cap\left(\bar{\Omega}_{2} \backslash \Omega_{1}\right)$. 
These theorems are extensively applied to many problems of various kinds; see $[1,4,8,9,11,12,14]$, for example. In $[2,17]$, the authors only dealt with modifications of the Guo-Krasnosel'skii fixed point theorem concerning cone expansion and compression of norm type, respectively. However, fixed point theorem concerning cone expansion and compression of order type to our knowledge has not been generalized or extended. In this paper, the fixed point theorems concerning cone expansion and compression of norm type and order type are both extended.

Lemma 1.2. Let $P$ be a cone in a real Banach space $E, \Omega$ a bounded open subset of $E$ with $\theta \in \Omega$, and $A: P \cap \bar{\Omega} \rightarrow P$ a completely continuous operator. If

$$
A x \neq \mu x
$$

for all $x \in P \cap \partial \Omega$ and $\mu \geq 1$, then the fixed point index

$$
i(A, P \cap \Omega, P)=1 \text {. }
$$

Lemma 1.3. Let $P$ be a cone in a real Banach space $E, \Omega$ a bounded open subset of $E$, and $A: P \cap \bar{\Omega} \rightarrow P$ a completely continuous operator. If

(i) $\inf _{x \in P \cap \partial \Omega}\|A x\|>0$ and

(ii) $A x \neq \mu x$ for all $x \in P \cap \partial \Omega$ and $\mu \in(0,1]$, then the fixed point index

$$
i(A, P \cap \Omega, P)=0 \text {. }
$$

Lemma 1.4. Let $P$ be a cone in a real Banach space $E, \Omega$ a bounded open subset of $E$, and $A: P \cap \bar{\Omega} \rightarrow P$ a completely continuous operator. Assume that there exists a $u_{0} \in P, u_{0} \neq \theta$ such that

$$
x-A x \neq \mu u_{0}
$$

for all $x \in P \cap \partial \Omega$ and $\mu \geq 0$, then the fixed point index

$$
i(A, P \cap \Omega, P)=0 .
$$

\section{Main results}

In this section, we present the main results of this paper.

Theorem 2.1. Let $P$ be a cone in a real Banach space $E, \Omega$ a bounded open subset of $E$ with $\theta \in \Omega$, and $A: P \cap \bar{\Omega} \rightarrow P$ a completely continuous operator. Assume that there exists another cone $P_{1}$ in another real Banach space $E_{1}$ and a homogeneous operator $B: P \rightarrow P_{1}$ with $\{B x \mid x \in P \cap \partial \Omega\} \subset P_{1} \backslash\{\theta\}$, such that

$$
B A x \leq B x, \quad \forall x \in P \cap \partial \Omega,
$$

this partial order is induced by the cone $P_{1}$ in $E_{1}$, then the fixed point index

$$
i(A, P \cap \Omega, P)=1 \text {. }
$$


Proof. If there exist $x_{0} \in P \cap \partial \Omega$ and $\lambda_{0} \geq 1$ such that $A x_{0}=\lambda_{0} x_{0}$, then $\lambda_{0}>1$. Therefore

$$
B\left(A x_{0}\right)=B\left(\lambda_{0} x_{0}\right)=\lambda_{0} B x_{0}>B x_{0},
$$

which contradicts (2.1). Hence the proof is finished by Lemma 1.2.

Theorem 2.2. Let $P$ be a cone in a real Banach space $E, \Omega$ a bounded open subset of $E$, and $A: P \cap \bar{\Omega} \rightarrow P$ a completely continuous operator. If

(i) $\inf _{x \in P \cap \partial \Omega}\|A x\|>0$ and

(ii) there exists another cone $P_{1}$ in another real Banach space $E_{1}$ and a homogeneous operator $B: P \rightarrow P_{1}$ with $\{B x \mid x \in P \cap \partial \Omega\} \subset P_{1} \backslash\{\theta\}$, such that

$$
B A x \geq B x, \quad \forall x \in P \cap \partial \Omega,
$$

this partial order is induced by the cone $P_{1}$ in $E_{1}$, then the fixed point index

$$
i(A, P \cap \Omega, P)=0 .
$$

Proof. If there exist $x_{0} \in P \cap \partial \Omega$ and $0<\lambda_{0} \leq 1$ such that $A x_{0}=\lambda_{0} x_{0}$, then $0<\lambda_{0}<1$. Therefore

$$
B\left(A x_{0}\right)=B\left(\lambda_{0} x_{0}\right)=\lambda_{0} B x_{0}<B x_{0},
$$

which contradicts (2.2). Hence the proof is finished by Lemma 1.3.

Theorem 2.3. Let $\Omega_{1}$ and $\Omega_{2}$ be two bounded open sets in $E$ such that $\theta \in \Omega_{1}$ and $\bar{\Omega}_{1} \subset \Omega_{2}$. Suppose that $A: P \cap\left(\bar{\Omega}_{2} \backslash \Omega_{1}\right) \rightarrow P$ is completely continuous. Assume that there exists two cones $P_{1}$ and $P_{2}$ in the Banach spaces $E_{1}$ and $E_{2}$ respectively, and homogeneous operators $B_{1}: P \rightarrow P_{1}$ with $\left\{B_{1} x \mid x \in\right.$ $\left.P \cap \partial \Omega_{1}\right\} \subset P_{1} \backslash\{\theta\}$ and $B_{2}: P \rightarrow P_{2}$ with $\left\{B_{2} x \mid x \in P \cap \partial \Omega_{2}\right\} \subset P_{2} \backslash\{\theta\}$. If one of the two conditions:

$\left(H_{1}^{*}\right) \quad B_{1} A x \leq B_{1} x, \forall x \in P \cap \partial \Omega_{1}$ and $\inf _{x \in P \cap \partial \Omega_{2}}\|A x\|>0, B_{2} A x \geq B_{2} x$, $\forall x \in P \cap \partial \Omega_{2}$;

$\left(H_{2}^{*}\right) \inf _{x \in P \cap \partial \Omega_{1}}\|A x\|>0, B_{1} A x \geq B_{1} x, \forall x \in P \cap \partial \Omega_{1}$ and $B_{2} A x \leq B_{2} x$, $\forall x \in P \cap \partial \Omega_{2}$;

is satisfied, then $A$ has at least one fixed point in $P \cap\left(\bar{\Omega}_{2} \backslash \Omega_{1}\right)$.

The proof is easy by Theorems 2.1 and 2.2 , and hence we omit it.

Remark 2.1. We claim that Theorem 2.3 is the extension of the fixed point theorem of cone expansion and compression of norm type. Indeed, if we take $B_{1} x=B_{2} x=\|x\|, \forall x \in P$, then $B_{1}\left(B_{2}\right): P \rightarrow \mathbb{R}^{+}$is a homogeneous operator and $\|x\| \neq 0, \forall x \in P \cap \partial \Omega_{1}$ or $x \in P \cap \partial \Omega_{2}$. Moreover $\inf _{x \in P \cap \partial \Omega_{1}}\|A x\|>0$ and $\inf _{x \in P \cap \partial \Omega_{2}}\|A x\|>0$ is satisfied naturally by $\theta \in \Omega_{1}$ and $\bar{\Omega}_{1} \subset \Omega_{2}$.

Theorem 2.4. Let $P$ be a cone in a real Banach space $E, \Omega$ a bounded open subset of $E$ with $\theta \in \Omega$, and $A: P \cap \bar{\Omega} \rightarrow P$ a completely continuous operator. 
Assume that there exists another cone $P_{1}$ in another real Banach space $E_{1}$ and an order-preserving operator $B: P \rightarrow P_{1}$, such that

$$
B A x \nsupseteq B x, \quad \forall x \in P \cap \partial \Omega,
$$

this partial order is induced by the cone $P_{1}$ in $E_{1}$, then the fixed point index

$$
i(A, P \cap \Omega, P)=1 \text {. }
$$

Proof. If there exist $x_{0} \in P \cap \partial \Omega$ and $\lambda_{0} \geq 1$ such that $A x_{0}=\lambda_{0} x_{0}$, then $A x_{0} \geq x_{0}$. Therefore

$$
B A x_{0} \geq B x_{0}
$$

which contradicts (2.3). Hence the proof is finished by Lemma 1.2.

Theorem 2.5. Let $P$ be a cone in a real Banach space $E, \Omega$ a bounded open subset of $E$, and $A: P \cap \bar{\Omega} \rightarrow P$ a completely continuous operator. Assumed that there exists another cone $P_{1}$ in another real Banach space $E_{1}$ and an order-preserving operator $B: P \rightarrow P_{1}$, such that

$$
B A x \not \leq B x, \quad \forall x \in P \cap \partial \Omega,
$$

this partial order is induced by the cone $P_{1}$ in $E_{1}$, then the fixed point index

$$
i(A, P \cap \Omega, P)=0 \text {. }
$$

Proof. If there exist $u_{0} \in P, u_{0} \neq \theta, x_{0} \in P \cap \partial \Omega$ and $\lambda_{0} \geq 0$, such that $x_{0}-A x_{0}=\lambda_{0} u_{0}$, then $A x_{0} \leq x_{0}$. Therefore

$$
B A x_{0} \leq B x_{0}
$$

which contradicts (2.4). Hence the proof is finished by Lemma 1.4.

Theorem 2.6. Let $\Omega_{1}$ and $\Omega_{2}$ be two bounded open sets in $E$ such that $\theta \in \Omega_{1}$ and $\bar{\Omega}_{1} \subset \Omega_{2}$. Suppose that $A: P \cap\left(\bar{\Omega}_{2} \backslash \Omega_{1}\right) \rightarrow P$ is completely continuous. Assume that there exists two cones $P_{1}$ and $P_{2}$ in the Banach spaces $E_{1}$ and $E_{2}$ respectively, and order-preserving operators $B_{1}: P \rightarrow P_{1}, B_{2}: P \rightarrow P_{2}$. If one of the two conditions:

$\left(H_{3}^{*}\right) \quad B_{1} A x \nsupseteq B_{1} x, \forall x \in P \cap \partial \Omega_{1}$ and $B_{2} A x \not \leq B_{2} x, \forall x \in P \cap \partial \Omega_{2}$;

$\left(H_{4}^{*}\right) \quad B_{1} A x \not \leq B_{1} x, \forall x \in P \cap \partial \Omega_{1}$ and $B_{2} A x \nsupseteq B_{2} x, \forall x \in P \cap \partial \Omega_{2}$; is satisfied, then $A$ has at least one fixed point in $P \cap\left(\bar{\Omega}_{2} \backslash \Omega_{1}\right)$.

The proof is easy by Theorems 2.4 and 2.5, and hence we omit it.

Remark 2.2. While using Guo-Krasnosel'skii fixed point theorems concerning cone expansion and compression of order type, we find that the partial order induced by the cone $P$ is difficult to check in a Banach space $E$. So we introduce another cone $P_{1}$ in another real Banach space $E_{1}$, the partial order induced by $P_{1}$ is easily satisfied. Obviously, if we take $B_{1} \equiv B_{2} \equiv \mathbf{I}$ (the identical mapping), then $B_{1}\left(B_{2}\right): P \rightarrow P$ is an order-preserving operator. So far, we realize that the fixed point theorems concerning cone expansion and compression of order type is a special case of Theorem 2.6, namely it is improved. 


\section{Applications}

In this section, we apply the results in Section 2 to the existence of multiple positive solutions for system of Hammerstein type integral equations given by

$$
\left\{\begin{array}{l}
\varphi(x)=\int_{G} k_{1}(x, y) f_{1}(y, \varphi(y), \psi(y)) d y, \\
\psi(x)=\int_{G} k_{2}(x, y) f_{2}(y, \varphi(y), \psi(y)) d y,
\end{array}\right.
$$

where $G$ is a bounded closed domain in $\mathbb{R}^{n}, k_{i}(x, y): G \times G \rightarrow \mathbb{R}^{+}$is a nonnegative continuous function, and $f_{i}(x, u, v): G \times \mathbb{R}^{+} \times \mathbb{R}^{+} \rightarrow \mathbb{R}^{+}$is a continuous function $(i=1,2)$. If we let

$$
A_{i}(\varphi, \psi)(x)=\int_{G} k_{i}(x, y) f_{i}(y, \varphi(y), \psi(y)) d y, \quad i=1,2,
$$

and $A(\varphi, \psi)=\left(A_{1}(\varphi, \psi), A_{2}(\varphi, \psi)\right)$, then (3.1) is equivalent to the fixed point of operator $A$.

Let the operator $K_{i}$ be defined as

$$
\left(K_{i} \varphi\right)(x)=\int_{G} k_{i}(x, y) \varphi(y) d y(x \in G), \quad i=1,2,
$$

and the spectral radiuses $r\left(K_{i}\right)>0(i=1,2) . f_{i}(x, u, v): G \times \mathbb{R}^{+} \times \mathbb{R}^{+} \rightarrow \mathbb{R}^{+}$ a continuous function and $k_{i}$ a nonnegative function imply that linear operator $K_{i}$ is complete continuous $(i=1,2)$. Evidently, operator $A_{i}$ is also complete continuous $(i=1,2)$. Then by the well-known Krein-Rutman Theorem [7], there exist continuous functions $g_{i}(x) \geq 0, g_{i}(x) \not \equiv 0$ such that

$$
\int_{G} k_{i}(y, x) g_{i}(y) d y=r\left(K_{i}\right) g_{i}(x), \quad \forall x \in G, i=1,2 .
$$

We make the following assumptions:

$\left(C_{1}\right)$ there exist continuous nonnegative function $a_{i}(x)(i=1,2)$, such that

$$
\begin{aligned}
k_{i}(x, y) & \geq a_{i}(x) k_{i}(\tau, y), \forall x, y, \tau \in G, \\
\delta_{i} & =\int_{G} a_{i}(x) g_{i}(x) d x>0,
\end{aligned}
$$

where $g_{i}(x)$ are determined by (3.2);

$\left(C_{2}\right)$ If one of the two conditions

$$
\lim _{u \rightarrow 0^{+}} \frac{f_{1}(x, u, v)}{u}>r^{-1}\left(K_{1}\right)
$$

hold uniformly for $x \in G, v \in \mathbb{R}^{+}$and

$$
\lim _{v \rightarrow 0^{+}} \frac{f_{2}(x, u, v)}{v}>r^{-1}\left(K_{2}\right)
$$

hold uniformly for $x \in G, u \in \mathbb{R}^{+}$is satisfied; 
$\left(C_{3}\right)$ Let

$$
\lim _{u \rightarrow+\infty} \frac{f_{1}(x, u, v)}{u}>r^{-1}\left(K_{1}\right)
$$

hold uniformly for $x \in G, v \in \mathbb{R}^{+}$and

$$
\lim _{v \rightarrow+\infty} \frac{f_{2}(x, u, v)}{v}>r^{-1}\left(K_{2}\right)
$$

hold uniformly for $x \in G, u \in \mathbb{R}^{+}$.

$\left(C_{4}\right)$ There exist a $r_{1}>0$ with $0<u+v<r_{1}$, such that

$$
f_{i}(x, u, v) \leq \lambda r_{1}(\forall x \in G),
$$

where $0<\lambda \leq\left(\left\|h_{1}\right\|+\left\|h_{2}\right\|\right)^{-1}, h_{i}(x)=\int_{G} k_{i}(x, y) d y,\left\|h_{i}\right\|=\max _{x \in G}\left|h_{i}(x)\right|$, $i=1,2$.

Theorem 3.1. Suppose that conditions $\left(C_{1}\right)-\left(C_{4}\right)$ hold. Then problem $(3.1)$ has at least two positive continuous solutions $\left(\varphi_{1}, \psi_{1}\right)$ and $\left(\varphi_{2}, \psi_{2}\right)$ satisfying

$$
0<\left\|\varphi_{1}\right\|+\left\|\psi_{1}\right\|<r_{1}<\left\|\varphi_{2}\right\|+\left\|\psi_{2}\right\| \text {. }
$$

Proof. Let $C(G)=\{\varphi \mid \varphi(x)$ is continuous on $\mathrm{G}\}, \mathrm{X}=C(G) \times C(G)$. The norm in $X$ is defined as $\|(\varphi, \psi)\|_{X}=\|\varphi\|+\|\psi\|$, and obviously $X$ is a Banach space. Let

$$
P_{i}=\left\{\varphi \in C(G) \mid \varphi(x) \geq 0, \int_{G} g_{i}(x) \varphi(x) d x \geq \delta_{i}\|\varphi\|\right\},
$$

where $\delta_{i}$ are determined by $(3.4)(i=1,2)$. It is easy to see that $P=P_{1} \times P_{2}$ is a cone in $X$. Let

$$
T(\varphi, \psi)=\left(r^{-1}\left(K_{1}\right) K_{1} \varphi, r^{-1}\left(K_{2}\right) K_{2} \psi\right) .
$$

Now we first show that the operator $A$ maps $P$ into $P$. In fact, for any $(\varphi, \psi) \in P$, by virtue of $(3.3)$ we get

$$
\begin{aligned}
\int_{G} g_{1}(x) A_{1}(\varphi, \psi)(x) d x & =\int_{G} g_{1}(x) d x \int_{G} k_{1}(x, y) f_{1}(y, \varphi(y), \psi(y)) d y \\
& \geq \int_{G} g_{1}(x) d x \int_{G} a_{1}(x) k_{1}(\tau, y) f_{1}(y, \varphi(y), \psi(y)) d y \\
& \geq \delta_{1} A_{1}(\varphi, \psi)(\tau), \forall \tau \in G,
\end{aligned}
$$

which implies $A_{1}(\varphi, \psi) \in P_{1}$. Similarly $A_{2}(\varphi, \psi) \in P_{2}$ and hence $A(\varphi, \psi) \in P$, namely, $A(P) \subset P$. Similarly $T(P) \subset P$. We take

$$
B(\varphi, \psi)=\int_{G} g_{1}(x) \varphi(x) d x,
$$


then $B$ maps $P$ into another cone $P_{1}=[0,+\infty)$ in a real Banach space $E_{1}=\mathbb{R}$ and evidently $B$ is an order-preserving and homogeneous operator. Let

$$
\Omega_{r}=\{(\varphi, \psi) \in X \mid\|\varphi\|+\|\psi\|<r\},
$$

then $\theta \in \Omega_{r}$.

Condition (3.5) implies that we can find a number $r_{0}$ with $0<r_{0}<r_{1}$ such that

$$
f_{1}(x, u, v) \geq r^{-1}\left(K_{1}\right) u, \quad \forall 0<u \leq r_{0} .
$$

Without loss of generality, we may assume that (3.5) hold in $\left(C_{2}\right)$ and $A$ has no fixed point on $\partial \Omega_{r_{0}}$. In virtue of (3.2) and (3.10), for any $(\varphi, \psi) \in \partial \Omega_{r_{0}} \cap P$, we get

$$
\begin{aligned}
& B A(\varphi, \psi)-B(\varphi, \psi) \\
= & \int_{G} g_{1}(x) A_{1}(\varphi(x), \psi(x)) d x-B(\varphi, \psi) \\
= & \int_{G} g_{1}(x) d x \int_{G} k_{1}(x, y) f_{1}(y, \varphi(y), \psi(y)) d y-\int_{G} g_{1}(x) \varphi(x) d x \\
= & r\left(K_{1}\right) \int_{G} g_{1}(y) f_{1}(y, \varphi(y), \psi(y)) d y-\int_{G} g_{1}(x) \varphi(x) d x \\
\geq & 0 .
\end{aligned}
$$

It is clear that $B A(\varphi, \psi)-B(\varphi, \psi) \neq 0$, thus $B A(\varphi, \psi)-B(\varphi, \psi)>0$. Hence for any $(\varphi, \psi) \in \partial \Omega_{r_{0}} \cap P$, such that

$$
B A(\varphi, \psi) \not \leq B(\varphi, \psi) \text {. }
$$

It follows from Theorem 2.5 that the fixed point index

$$
i\left(A, \Omega_{r_{0}} \cap P, P\right)=0 .
$$

From $(3.7)$ in $\left(C_{3}\right)$, then there exist positive numbers $\varepsilon$ and $b_{1}$, such that

$$
f_{1}(x, u, v) \geq\left(r^{-1}\left(K_{1}\right)+\varepsilon\right) u-b_{1}, \quad \forall x \in G, u \geq 0, v \geq 0 .
$$

We put

$$
R>\max \left\{r_{1}, \frac{2 b_{1} \int_{G} g_{1}(y) d y}{\varepsilon r\left(K_{1}\right) \delta_{1}}\right\}
$$

and let

$$
\Omega_{R}=\{(\varphi, \psi) \in X \mid\|\varphi\|+\|\psi\|<R\} .
$$

Without loss of generality, we may assume that $A$ has no fixed point on $\partial \Omega_{R}$. For any $(\varphi, \psi) \in \partial \Omega_{R} \cap P$, we have $\|\varphi\|+\|\psi\|=R$. Suppose $\|\varphi\| \geq \frac{R}{2}$, because the proof is similar when $\|\psi\| \geq \frac{R}{2}$. In virtue of (3.12) and (3.2) we get 


$$
\begin{aligned}
& B A(\varphi, \psi)-B(\varphi, \psi) \\
= & \int_{G} g_{1}(x) A_{1}(\varphi(x), \psi(x)) d x-B(\varphi, \psi) \\
= & \int_{G} g_{1}(x) d x \int_{G} k_{1}(x, y) f_{1}(y, \varphi(y), \psi(y)) d y-\int_{G} g_{1}(x) \varphi(x) d x \\
= & r\left(K_{1}\right) \int_{G} g_{1}(y) f_{1}(y, \varphi(y), \psi(y)) d y-\int_{G} g_{1}(x) \varphi(x) d x \\
\geq & r\left(K_{1}\right)\left(r^{-1}\left(K_{1}\right)+\varepsilon\right) \int_{G} g_{1}(y) \varphi(y) d y-b_{1} \int_{G} g_{1}(y) d y-\int_{G} g_{1}(x) \varphi(x) d x \\
= & \varepsilon r\left(K_{1}\right) \int_{G} g_{1}(y) \varphi(y) d y-b_{1} \int_{G} g_{1}(y) d y \\
\geq & \varepsilon r\left(K_{1}\right) \delta_{1}\|\varphi\|-b_{1} \int_{G} g_{1}(y) d y>0 .
\end{aligned}
$$

Hence for any $(\varphi, \psi) \in \partial \Omega_{R} \cap P$, such that

$$
B A(\varphi, \psi) \not B B(\varphi, \psi) \text {. }
$$

It follows from Theorem 2.5 that the fixed point index

$$
i\left(A, \Omega_{R} \cap P, P\right)=0 .
$$

Without loss of generality, we may assume that $A$ has no fixed point on $\partial \Omega_{r_{1}}$. For any $(\varphi, \psi) \in \partial \Omega_{r_{1}} \cap P$, then $\|\varphi\|+\|\psi\|=r_{1}$. By (3.9), we get

$$
A_{i}(\varphi, \psi)(x) \leq \lambda r_{1} \int_{G} k_{i}(x, y) d y=\lambda r_{1} h_{i}(x), \quad(i=1,2),
$$

then $\left\|A_{i}(\varphi, \psi)\right\| \leq \lambda r_{1}\left\|h_{i}\right\|$. Therefore,

$$
\|A(\varphi, \psi)\|_{X} \leq \lambda r_{1}\left(\left\|h_{1}\right\|+\left\|h_{2}\right\|\right) \leq r_{1}=\|(\varphi, \psi)\|_{X} .
$$

By taking $B x=\|x\|_{X}, \forall x \in P$ in Theorem 2.1, we see that the fixed point index

$$
i\left(A, \Omega_{r_{1}} \cap P, P\right)=1 \text {. }
$$

It is clear that $\bar{\Omega}_{r_{0}} \subset \Omega_{r_{1}}, \bar{\Omega}_{r_{1}} \subset \Omega_{R}$. We see that (3.11), (3.13) and (3.14) imply by virtue of $\left(G_{2}\right)$ in Lemma 1.1 the fixed point index $i\left(A,\left(\Omega_{R} \backslash \Omega_{r_{1}}\right) \cap\right.$ $P, P)=-1, i\left(A,\left(\Omega_{r_{1}} \backslash \Omega_{r_{0}}\right) \cap P, P\right)=1$. Hence, $\left(G_{6}\right)$ in Lemma 1.1 implies that $A$ has at least two positive continuous solutions $\left(\varphi_{1}, \psi_{1}\right)$ and $\left(\varphi_{2}, \psi_{2}\right)$ satisfying

$$
0<\left\|\varphi_{1}\right\|+\left\|\psi_{1}\right\|<r_{1}<\left\|\varphi_{2}\right\|+\left\|\psi_{2}\right\| \text {. }
$$

This completes the proof.

Remark 3.1. In $[13,14]$, the authors only obtained the existence of positive solutions to systems of nonlinear Hammerstein type integral equations. However, the existence of multiple positive solutions is obtained here and the main method used in the proof is essentially different from the literature $[13,14]$. 
Acknowledgements. The authors are grateful to the referee whose comments have led to a number of significant improvements of the paper.

\section{References}

[1] R. P. Agarwal, S. R. Grace, and D. ORegan, Semipositone higher-order differential equations, Appl. Math. Lett. 17 (2004), 201-207.

[2] D. R. Anderson and R. I. Avery, Fixed point theorem of cone expansion and compression of functional type, J. Difference Equations Appl. 8 (2002), 1073-1083.

3] K. Deimling, Nonlinear Functional Analysis, Springer-Verlag, New York, 1985.

[4] P. Eloe and J. Henderson, Positive solutions for $(n-1,1)$ conjugate boundary value problems, Nonlinear Anal. TMA 28 (1997), 1669-1680.

[5] D. Guo and V. Lakshmikantham, Nonlinear Problems in Abstract Cones, Academic Press, San Diego, 1988.

[6] D. Guo and J. Sun, Nonlinear Integral Equations, Shandong Press of Science and Technology, Jinan, 1987.

[7] M. G. Krein and M. A. Rutman, Linear operators leaving invariant a cone in a Banach space, Transl. Amer. Math. Soc. 10 (1962), 199-325.

[8] R. Ma and H. Wang, On the existence of positive solutions of fourth-order ordinary differential equations, Appl. Anal. 59 (1995), 225-231.

[9] _ Positive solutions of nonlinear three-point boundary-value problems, J. Math. Anal. Appl. 282 (2003), 232-240.

[10] J. Sun and X. Liu, Computation for topological degree and its applications, J. Math. Anal. Appl. 202 (1996), 785-796.

[11] Z. Yang, Existence and nonexistence results for positive solutions of an integral boundary value problem, Nonlinear Anal. 65 (2006), 1489-1511.

[12] _ Positive solutions of a second-order integral boundary value problem, J. Math. Anal. Appl. 321 (2006), 751-765.

[13] _ Nontrivial solutions of systems of nonlinear Hammerstein integral equations and applications, Mathematica Acta Scientia, 26A (2006), no. 2, 233-240.

[14] Z. Yang and Donal O'Regan, Positive solvability of systems of nonlinear Hammerstein integral equations, J. Math. Anal. Appl. 311 (2005), 600-614.

[15] Z. Yang and J. Sun, Positive solutions of boundary value problems for systems of nonlinear second order ordinary differential equations, Acta Mathematica Sinica, 47 (2004), 111-118.

[16] E. Zeidler, Nonlinear Functional Analysis and its Applications I: Fixed-Point Theorems, Springer-Verlag, New York, 1986.

[17] G. Zhang and J. Sun, A generalization of the cone expansion and compression fixed point theorem and applications, Nonlinear Anal. 67 (2007), 579-586.

FENG WANG

School of Mathematics and Physics

JiAngsu Polytechnic University

Changzhou 213164, P. R. China

E-mail address: fengwang188@163.com

FANG ZHANG

School of Mathematics and Physics

Jiangsu Polytechnic University

Changzhou 213164, P. R. China

E-mail address: zhangfang@tom.com 